\title{
THE TRIANGULATION PROBLEM AND ITS ROLE IN ANALYSIS
}

\author{
STEWART S. CAIRNS
}

1. Introduction. The triangulation problem is fundamental in the topology of manifolds and is closely related to certain methods employed in analysis. The results to date are, perhaps, of greater interest from the viewpoint of connections between topology and differential geometry (or other branches of analysis) than from a purely topological viewpoint. Most of the published work on this problem has appeared during approximately the last fifteen years, save for well known results in two dimensions. A brief discussion of a 2-dimensional result and its role in proving a theorem of analysis (adapted from Osgood's Funktionentheorie [31 ${ }^{1}$ ). may throw light on the problem and a certain class of applications.

Let $B$ denote a simple closed curve in the $(x, y)$-plane, and let $R$ denote its interior. It will be assumed that $B$ is differentiable in the sense that some neighborhood of any point on $B$ can be represented by giving $y$ (or $x$ ) as a single-valued function of $x$ (or $y$ ) with a continuous first derivative. Let the entire $(x, y)$-plane be subdivided into squares by the lines

$$
x=m \delta, \quad y=n \delta,
$$

$$
m, n=0, \pm 1, \pm 2, \cdots,
$$

where $\delta$ is a positive number so small that a circle of radius $3 \delta$ about any point of $B$ cuts from $B$ a single arc, any two tangents to which form an angle less than $\pi / 6$. If, in or on the boundary of one of the squares determined by (1.1), $B$ is (1) parallel at some point to an axial direction and (2) meets an edge, $\beta$, parallel to that same direction, then let the two squares incident with $\beta$ be amalgamated into a single rectangular region. The amalgamated region cuts from $B$ a single arc with end points on the sides perpendicular to $\beta$. This arc divides the rectangle into two parts, one in $R$ and one outside. The part inside $R$ will be a 2-cell of the subdivision of $(R+B)$. Each of the squares which meets $(R+B)$ and is not involved in such an amalgamation has in its interior just a 2-cell of $R$, to be reckoned as a 2-cell of the subdivision. The 1-cells of the subdivision consist of (1) the edges entirely in $R$ of the squares determined by (1.1), (2) the

An address delivered before the New York meeting of the Society on April 26, 1946, by invitation of the Committee to Select Hour Speakers for Eastern Sectional Meetings; received by the editors May 2, 1946.

${ }^{1}$ Numbers in brackets refer to the Bibliography at the end of the paper. 
segments in $R$ of those edges which are met by $B$, and (3) the arcs cut from $B$ by amalgamated rectangles and by unmodified squares of the set (1.1). The 0-cells are, of course, the end points of the 1-cells just defined.

Osgood employed the above subdivision to prove rigorously, among other results, the 2-dimensional case of Green's Theorem, which can be expressed in the form

$$
\iint_{R}\left(\frac{\partial X}{\partial y}-\frac{\partial Y}{\partial x}\right) d S=-\int_{B}(X \cos \tau+Y \sin \tau) d s
$$

where (1) $(X, Y)$ is a vector field with continuous first partial derivatives, (2) $B$ is oriented counterclockwise, and (3) $\tau$ is the inclination of the directed tangent line to $B$. The theorem is invariant under euclidean transformations of coordinates. After a suitable rotation of axes, it is easy to prove it for a typical 2-cell of the subdivision. This gives the result in the small. Its proof in the large is obtained by summing the identities for all the 2-cells of the subdivisions. Each inner 1-cell is common to the boundaries of two 2-cells and is oppositely oriented on these boundaries. Hence the contributions from all inner 1-cells add up to zero, and the desired result follows.

Kellogg [28] directly generalized the above procedure to three dimensions, and the writer $[5,18]$ carried through such a generalization in $n$ dimensions.

The word triangulation suggests a subdivision of a curved surface, or a plane region, into (curvilinear) triangles. However, cellular subdivisions of the sort just described are also referred to as triangulations, as are the higher-dimensional generalizations defined in \$4.

The above work reveals a cellular subdivision as a useful tool in proving a theorem of analysis. In general, such applications require not merely a knowledge of the triangulability of the region or locus in question, but require a subdivision thereof into cells possessing special properties.

2. Manifolds of various classes. Intrinsic definition. The most fundamental and interesting triangulation problems relate to manifolds. A topological m-manifold is a connected topological space which can be covered by a denumerable set of neighborhoods, called $m$-cells, each of which is homeomorphic to the interior of an $(m-1)$-dimensional hypersphere in euclidean $m$-space.

The manifolds of differential geometry and analysis are subject to differentiability conditions, and the most general triangulation theorems to date have been proved only with the aid of such conditions. 
Some of the following definitions are more general than necessary for immediate purposes. They are so stated partly for their own interest and partly for later reference.

Let $R^{m}$ be any set of points in one-to-one correspondence with a point set in a euclidean $m$-space, $E^{m}$. By identifying the points of $R^{m}$ with their respective images, we define continuity, open sets, and so on, on $R^{m}$. Thus a set on $R^{m}$ is open if and only if the corresponding set is open relative to $E^{m}$. Let rectangular cartesian coordinates be introduced in $E^{m}$, and assign to each point of $R^{m}$ the coordinates of its image. We thus obtain a coordinate system $(u)$ with $R^{m}$ for its domain. The inner domain of $(u)$ will mean the set of inner points of $R^{m}$. It may be vacuous.

If the domains of two coordinate systems $(u) \equiv\left(u_{1}, \cdots, u_{m}\right)$ and $(v) \equiv\left(v_{1}, \cdots, v_{n}\right)$ have common points, then the transformation between $(u)$ and $(v)$ will mean the correspondence

$$
v_{i}=v_{i}(u)
$$

$$
(i=1, \cdots, n)
$$

giving the $(v)$-coordinates in terms of the $(u)$-coordinates for every point common to the two domains.

Let $D$ be any open subset of the domain of $(u)$. Then a function $f(u)$ will be called of class $C^{r}$ on $D$, where $r$ is a non-negative integer, if $f(u)$ and all its derivatives of orders not greater than $r$ are defined and continuous on $D$. It will be called of class $C^{r}$ on $^{2} \bar{D}$ if, in addition, $f(u)$ and all its derivatives of orders not greater than $r$ have continuous limiting values ${ }^{3}$ on $\bar{D}-D$. If $f(u)$ is of class $C^{r}$ on $D$ (or $\bar{D}$ ) for every positive integer $r$, we shall refer to it as being of class $C^{\infty}$ on $D$ (or $\bar{D}$ ). A function of class $C^{\omega}$ on $D$ means one which is analytic on $D$. If, in addition, it is of class $C^{\infty}$ on $\bar{D}$, it is said to be of class $C^{\omega}$ on $\bar{D}$.

We shall say that the transformation (2.1) between two coordinate systems $(u) \equiv\left(u_{1}, \cdots, u_{m}\right)$ and $(v) \equiv\left(v_{1}, \cdots, v_{m}\right)$ is of class $C^{r}$, $r \in(0,1,2, \cdots, \infty, \omega)$, if $(1)$ the inner domains of $(u)$ and $(v)$ have a nonvacuous intersection, $D,(2)$ the correspondence defined by (2.1), interpreted as a mapping of part of $(u)$-space into (v)-space, is a homeomorphism, (3) each of the functions $v_{i}(u)$ is of class $C^{r}$ on $D$, and (4) in case $r>0$, the jacobian of the transformation is nowhere zero on $D$ (or, in case the domains of $(u)$ and $(v)$ are closed, on $\bar{D}$ ).

2 The symbol for a point set, modified by a bar, denotes the closure of the set.

3 Whitehead [13] restricts "differentiable functions" on a closed set, as $\bar{D}$, to those which can be differentiably extended to an open neighborhood of the closed set. $\mathrm{He}$ makes a similar restriction with respect to the "non-degeneracy" (in terms of the rank of a jacobian matrix) of a mapping of a closed set. The merits of these restrictions are discussed later. 
Consider the totality of coordinate systems $\left(x_{1}, \cdots, x_{m}\right)$ whose domains are $m$-cells on the topological $m$-manifold $M$. Suppose that, for some value $r \in(0,1, \cdots, \infty, \omega)$, there exists a subset, $S$, of these coordinate systems such that (1) every point of $M$ is on the domain of at least one of the systems $S$ and (2) every transformation between any two of the systems $S$ is of class $C^{r}$. Under these conditions, we shall say that $M$ is of class $C^{r}$ in terms ${ }^{4}$ of $S$. If $r>0$, then $M$ is said to be differentiable in terms of $S$, and if $r=\omega$, it is referred to as analytic in terms of $S$.

3. Manifolds in euclidean spaces. By an m-manifold of class $C^{k}$ $(k=1,2, \cdots, \infty, \omega)$ in $E^{n}$ is meant a set of points, $M$, in $E^{n}$, any point $P$ of which has an $m$-cell neighborhood on $M$ which can be defined by giving $(n-m)$ of the $y^{\prime}$ s as class $C^{k}$ functions of the remaining $y$ 's. These remaining $y^{\prime}$ s can then be interpreted as local coordinates on the neighborhood of $P$ in question. In terms of such local coordinates, $M$ also satisfies the intrinsic definition ( $\$ 2$ ) of an $m$-manfold of class $C^{k}$.

It was natural to inquire whether the intrinsically defined manifolds of class $C^{k}(k>0)$ are more general in their topological structure than manifolds of class $C^{k}$ in euclidean spaces. Whitney [40] answered this question in the negative, by showing that every manifold of class $C^{k}$, intrinsically defined, is homeomorphic to a manifold of class $C^{k}$ in a euclidean space. His results include a number of valuable properties, which we proceed to set forth, in so far as they are relevant to the present discussion.

Let $M$ be an $m$-manifold of class $C^{k}$ in terms of a set $S$ of local coordinate systems. Suppose, for some $n>m$, that it is possible to define $n$ functions of position $\left(y_{1}, \cdots, y_{n}\right)$ over the entire manifold $M$ in such a way that, for some positive integer, $\kappa,(1)$ the $y$ 's are continuous functions of the local coordinates $S,(2)$ all their derivatives of orders $(1, \cdots, \kappa)$ are defined and continuous in terms of each of the coordinate systems $S$ throughout its domain, (3) the jacobian matrix $\left\|\partial y_{i} / \partial x_{j}\right\|(i=1, \cdots, n ; j=1, \cdots, m)$ is of rank $m$ on the domain of each system $\left(x_{1}, \cdots, x_{m}\right)$ of $S$, and (4) the same set $\left(y_{1}, \cdots, y_{n}\right)$ of values is not assumed at two different points of $M$.

${ }^{4}$ It is important to note that for a manifold to be of class $C^{r}$ does not necessarily imply a structural restriction, since the class is defined as a property of a set of local coordinate systems. According to one of the important theorems quoted below $(\S 3(B))$, there is no topological distinction between manifolds of class $C^{1}$ and those of any class $C^{r}(r>1)$. The question whether manifolds of class $C^{0}$ (that is, topological manifolds in general) are topologically equivalent to those of class $C^{1}$ is considered in $\$ 14$. 
(A) If $\left(y_{1}, \cdots, y_{n}\right)$ are now interpreted as rectangular cartesian coordinates in a euclidean space $E^{n}$, then the functions just described afford a nonsingular parametric representation $M^{*}$ of $M$ in $E^{n}$, with the coordinate systems $S$ serving as local parameters. Such a representation is referred to as a class $C^{*}$ map or imbedding of $M$. In the above description, we necessarily have $\kappa \leqq k$, if $M$ is of class $C^{k}$ (but not of class $\left.C^{k+1}\right)$ in terms of $S$.

(B) In the preceding statement, it is easy to show, with the aid of the implicit function theorem, that the parameters can be locally eliminated, in which case $M^{*}$ is locally represented by giving $(n-m)$ of the $y^{\prime}$ s as functions of the remaining $m$, and these functions are of class $C^{*}$. They might possibly also be of class $C^{\lambda}$ for some value $\lambda>k$. Whitney's work [40] included a proof that if $M$ is of class $C^{k}, k \in(1, \cdots, \infty)$, then it has a class $C^{k}$ imbedding, $M^{*}$, in $E^{2 m+1}$, and it can further be required that $M^{*}$ be of arbitrarily high class (analytic for example) in terms of the euclidean metric of $E^{2 m+1}$.

Among the implications of the last statement, it may be noted that differentiable manifolds are no more general in their topological structure than analytic manifolds. In other words, for manifolds of class $C^{r}$ $(r>0)$, topological properties depend in no way on the class number $r$. If $M$ is analytic, it is still not known whether it can be analytically mapped into a manifold in an $E^{n}$; though it can, as just stated, be mapped in class $C^{\infty}$ fashion into an analytic manifold in $E^{n}$.

It is possible to establish [27], very briefly and simply, a weaker imbedding theorem, to the effect merely that if $M$ is of class $C^{k}$, for any $k \in(1,2, \cdots, \infty)$, then $M$ has a class $C^{k}$ imbedding in a euclidean space of sufficiently high dimensionality.

4. The general triangulation problem. The complexes with which this discussion is concerned are cellular complexes, defined in the spirit of euclidean geometry rather than in a more abstract manner. These complexes represent the most general triangulated spaces, in the terminology of the present treatment. The following definitions are adapted from Alexandroff and Hopf [16].

A euclidean $n$-space $E^{n}$ is separated by an $(n-1)$-dimensional plane into two parts, the closure of either of which is called a half-space. A convex cell is a bounded subset of $E^{n}$ representable as the common part of a finite number of half-spaces..$^{5}$ For any such cell, $s$, there exists a number $r \in(0,1, \cdots, n)$ such that $s$ lies in some $r$-plane, $E^{r}$, but not in any $(r-1)$-plane. This number is called the dimensionality of $s$, which is referred to as a convex $r$-cell $s_{r}$. Such a cell $s_{r}$ is the

\footnotetext{
5 Cells are thus defined as closed sets, contrary to the usage in $\$ \S 1-3$ above.
} 
closure of an open region in $E^{r}$, called the interior of the cell. The boundary of $s_{r}\left(s_{r}\right.$ minus its interior) falls uniquely into a finite set of convex $(r-1)$-cells, called the bounding $(r-1)$-cells of $s_{r}$. Their bounding $(r-2)$-cells are called the bounding $(r-2)$-cells of $s_{r}$, and so on. The bounding 0 -cells are also called vertices. A convex $r$-cell with just $(r+1)$ vertices is an $r$-simplex.

A finite or denumerable set of convex cells in $E^{n}$ forms a rectilinear complex if (1) each bounding cell of a member of the set belongs to the set, (2) the intersection of any two cells of the set is a common bounding cell, ${ }^{6}$ and (3) no point is vertex of infinitely many cells of the set. A rectilinear complex $K$ is described as simplicial if all its cells are simplexes. It is referred to as locally finite if each of its points has a neighborhood which intersects at most a finite number of cells of the complex. The set of all points of $E^{n}$ each on one of the cells of a locally finite rectilinear complex is called a euclidean polyhedron.

Any topological image of a locally finite rectilinear complex $K$ is called a complex, and the images of the cells of $K$ are called the cells of the complex. Thus an $n$-cell is defined as a topological image of the closure of a finite convex region of $E^{n}$.

Any topological space will be referred to as locally polyhedral if it can be covered with a denumerable set of neighborhoods each homeomorphic with a neighborhood on some euclidean polyhedron.

The general triangulation PRoblem. Is it possible to subdivide every locally polyhedral space into the cells of a complex?

Otherwise expressed, the problem is to discover whether a topological space with the local structure of a complex necessarily has such a structure in the large. Failing this, it is of interest to discover necessary and sufficient conditions (either or both) that a locally polyhedral space be triangulable. The triangulation problem can be solved, with the aid of well known theorems, for locally polyhedral spaces of dimensionality 2 (or less), but it has not yet been solved, in the absence of differentiability conditions, for the general locally polyhedral space (or even for the general manifold) of any higher dimension.

The triangulation problem for manifolds is of basic importance in the more general triangulation problem, by virtue of the following easily proved result.

\section{(A) Any locally polyhedral space $\Pi$ can be expressed as a sum}

- For the sake of this definition, the vacuous set is regarded as a bounding cell (of dimensionality -1 ) of every cell, and an $r$-cell is counted among its own bounding cells. 


$$
\Pi=\sum M_{i}^{m} \quad(m=0,1, \cdots ; i=1,2, \cdots)
$$

where the $M_{i}^{m}$ are distinct topological $m$-manifolds such that, given any pair $\left(M_{i}^{m}, M_{j}^{n}\right)$, either the intersection $M_{i}^{m} \cdot \bar{M}_{j}^{n}$ is vacuous or else $n>m$ and $M_{i}^{m}$ is on $\bar{M}_{i}{ }^{n}$.

In other words, a locally polyhedral space is built up of manifolds with incidence relations analogous to those of the cells of a complex. This does not mean that the triangulability of topological manifolds would imply the triangulability of locally polyhedral spaces in general; for it is not certain that a triangulation of the boundary of a manifold could be extended over the manifold, even if both should be proved triangulable.

5. Significance of the problem. The manifolds whose topology was studied by Poincaré were essentially the same as our differentiable manifolds, although the present definitions had not been made at the time. Poincaré introduced the cellular complexes of combinatorial topology as a device for dealing numerically with certain problems which presented difficulty for his manifolds. It was, however, not obvious that the results of the combinatorial theory were directly applicable to these manifolds, and the entire development of combinatorial topology was carried out with no assurance of such applicability until the triangulation problem was solved for differentiable manifolds.

The triangulation problem is thus basic in the relationship between point theoretic and combinatorial topology. Its solution, however, even if carried through in the most general case, would not suffice to lay a solid foundation for a theory of topological manifolds from the combinatorial viewpoint. In comparing the point theoretic and combinatorial methods, it is seen that analogous roles are played by homeomorphisms in the former theory and by combinatorial equivalence in the latter. Two complexes are combinatorially equivalent if they have subdivisions which are isomorphic with respect to incidence relations. A general triangulation theorem for manifolds would thus need to be supplemented by a theorem regarding the combinatorial equivalence of two triangulations of the same manifold; or, what amounts to the same thing, of a pair of homeomorphic manifolds.

As brought out in later sections, adequate triangulation and combinatorial equivalence theorems have now been proved for differentiable manifolds, so that the results of combinatorial topology are now directly applicable to the spaces for the sake of which the theory was initiated. 
In the case of topological manifolds, and other locally polyhedral spaces, much work now done with the aid of approximations by complexes could probably be more readily accomplished with the aid of appropriate triangulation and equivalence theorems. However, the difficulties presented by these more general cases are of an entirely different order from those encountered under hypotheses of differentiability.

Aside from the primarily topological aspects of these problems, there exist many possibilities, some of which have already been realized, for the employment of triangulation theorems in analysis. Analysis and differential geometry are frequently concerned with spaces (algebraic or analytic varieties for example) defined by equations, or else with differentiable (analytic) manifolds of the sort defined in $\$ \$ 2$ and 3 above, and also in Veblen and Whitehead's book [37] on the foundations of differential geometry. The homology theory of combinatorial topology, for example, is of frequent applicability and can be carried over to the spaces in question with the aid of triangulations. Such considerations are touched upon in a later section of this paper.

A large class of applications of cellular subdivisions and related methods can be grouped into (1) the extension of local results to results in the large (cf. $\$ 1$ above) and (2) the extension of theorems proved for topological spaces subject to various restrictions, to apparently less restricted, but topologically equivalent, spaces. The significance of the foregoing remark can, fortunately, be illustrated by results in the literature, for the triangulation theorems thus far obtained have received applications of both of the types just mentioned.

We proceed, in $\S \S 6-11$, to give an account of research thus far published with a direct bearing on the triangulation problem. This account lays a foundation for a more detailed discussion of the role of the problem in analysis and of various ramifications of the problem.

6. Triangulation theorems in the literature. Consider a locally polyhedral space, $\Pi$, expressed as a sum of topological manifolds as in $\$ 4(\mathrm{~A})$. By the coordinate systems on $\Pi$, we mean the set of all coordinate systems $(x) \equiv\left(x_{1}, \cdots, x_{n}\right) \quad(n=1,2, \cdots)$ whose domains are (closed) $n$-cells on $\Pi$, each inner domain being on one of the manifolds $M_{i}{ }^{n}$. We will say that $\Pi$ is piecewise of class $C^{r}$ in terms of $S$ $(r=0,1, \cdots, \infty, \omega)$, where $S$ is a subset of these coordinate systems, if two conditions are fulfilled. The first is that $M_{i}{ }^{n}(n=1,2, \cdots$; $i=1,2, \cdots)$ be of class $C^{r}$ in terms of the subset of the systems $S$ consisting of all those whose inner domains are on $M_{i}{ }^{n}$. The second 
condition relates to coordinate systems on incident manifolds. Suppose $M_{i}{ }^{m} \subset \bar{M}_{j}{ }^{n}$ (cf. $\S 4(\mathrm{~A})$ ), and let $(x)$ and $(y)$ be systems of the set $S$ with inner domains on $M_{i}^{m}$ and $M_{j}^{n}$ respectively. We then require (1) that if the domains of $(x)$ and $(y)$ intersect, their intersection shall be the closure of an open set, $D$, relative to $M_{i}{ }^{m}$, (2) that the $y$ 's be class $C^{r}$ functions of the $x$ 's on $\bar{D}$, and (3) in case $r>0$, that the jacobian matrix $\left\|\partial y_{i} / \partial x_{j}\right\|$ be of rank $m$ on $\bar{D}$. We use the term piecewise differentiable as equivalent to piecewise of class $C^{1}$.

The most general triangulation theorem thus far established, so far as the writer is aware, is the following:

TRIANGULATION THEOREM. Every piecewise differentiable locally polyhedral space is triangulable.

This is the culmination of a series of results which appeared from time to time in the literature. A brief chronological outline of published material bearing directly on the problem is as follows:

(1) A proof [1] by van der Waerden of the triangulability of algebraic varieties.

(2) An abstract [2] by the writer, presenting triangulation theorems for (a) a region of euclidean $n$-space bounded by a finite number of differentiable manifolds, (b) a differentiable manifold in a euclidean space, and (c) a region of euclidean 3-space bounded by a finite number of piecewise differentiable 2 -manifolds. This abstract represented work done on a doctoral thesis at Harvard, under Professor Marston Morse, and the writer's later research on the subject can be regarded as an outgrowth of that work.

(3) A proof [3], outlined by Lefschetz in his colloquium lectures on topology, of the triangulability of analytical varieties.

(4) An article [4] by Koopman and Brown, in which (a) it was stated that, while van der Waerden's proof is valid for algebraic varieties, it cannot be extended to analytic varieties in general, (b) it was asserted that Lefschetz' discussion [3] was incomplete, and (c) an independent proof was given of the triangulability of analytic varieties.

(5) A paper [5] giving in detail the proof of the first triangulation theorem mentioned under (2) above.

(6) A detailed proof [6] of the triangulability of analytic loci by Lefschetz and Whitehead along the lines of the outlined proof in Lefschetz' book [3].

(7) A proof [7], by the writer, of the triangulability of a regular $r$-locus in a euclidean space. Such a locus has the same sort of definition as the piecewise differentiable locally polyhedral space. The pa- 
per employed a superfluous imbedding assumption, later shown to involve no essential loss of generality. Incidentally, it may be noted that the theorem of this paper includes all previously established triangulability results.

(8) An attack [8] by Nöbeling on the general triangulation problem for manifolds and on the problem of showing, if true, that any two triangulations of homeomorphic manifolds have equivalent subdivisions; that is, subdivisions isomorphic with respect to incidence relations of cells. The attack appeared successful, and its success would have afforded a foundation (cf. $\$ 5$ above) for a complete development of a combinatorial theory of topological manifolds. However, essential errors were noted, after the article was published, and were pointed out, for example, by Seifert [9] in a brief review of the paper. These two basic problems still remain unsolved in the general case.

(9) A proof [10] by the writer of the triangulability of the differentiable manifold. The purpose of this proof was to apply the triangulation methods and results of an earlier paper [7] explicitly to the manifolds of class one (or class $C^{1}$ ) as defined, for example, by Veblen and Whitehead [37], by Hodge [27], and in \$4 above.

(10) Papers $[11,12]$ by Brouwer and Freudenthal, respectively, on the triangulation of differentiable manifolds. These papers were written without knowledge of other publications on the subject.

(11) A treatment [13] by Whitehead, based partly on the writer's work, of triangulation theorems and related questions for differentiable loci.

(12) The writer's establishment [14] of the triangulability of piecewise differentiable locally polyhedral spaces.

7. Certain local properties of imbedded manifolds. It is natural, in the case of euclidean polyhedra (see $\$ 4$ above), to employ rectilinear methods of subdivision. These methods have the advantage, basic in the development of combinatorial topology, that any two rectilinear triangulations of the same euclidean polyhedron have isomorphic rectilinear subdivisions (cf. §5), a property not known to hold for more general triangulations.

The most straightforward methods $[7,10,13]$ of triangulation for differentiable loci are extensions and adaptations of rectilinear simplicial subdivisions. These methods depend for their success upon the manner in which a differentiable $m$-manifold can be locally approximated by inscribed $r$-simplexes $(r=1,2, \cdots, m)$.

Let $M$ denote a closed differentiable $m$-manifold in $E^{n}$. In view of Whitney's results, $M$ has all the generality, when proving its triangu- 
lability, of an intrinsically defined ${ }^{7}$ differentiable manifold. Let $(y) \equiv\left(y_{1}, \cdots, y_{n}\right)$ be a rectangular cartesian coordinate system in $E^{n}$. The notation $Y^{i}\left(i=1,2, \cdots, N=C_{n, m}\right)$ will be employed for the various combinations $Y^{i}=\left(y_{i_{1}}, \cdots, y_{i_{m}}\right)$ of the $y^{\prime}$ 's in sets of $m$. The coordinate $m$-plane of the set $Y^{i}$ will be referred to as the $Y^{i}$-plane. The projection of a point (or a set of points) on the $Y^{i}$-plane is obtained by equating to zero all the $y^{\prime}$ s not in the set $Y^{i}$ and is referred to as the $Y^{i}$-projection. If $V$ is an $m$-dimensional volume on the tangent $m$-plane to $M$ at $P$, and $V^{i}$ is the $Y^{i}$-projection of $V$, then the ratios $\gamma^{i}=V^{i} / V(i=1, \cdots, N)$ are, numerically, generalized direction cosines of $M$ at $P$. The sum of their squares is unity [7], so that the largest of them at any point $P$ on $M$ is at least $1 / N^{1 / 2}$. Let $D^{i}$ denote the subset of $M$ on which $\gamma^{i}>1 / 2 N^{1 / 2}$. Then $\left(D^{1}, \cdots, D^{N}\right)$ are an overlapping set of open regions covering $M$. On $D^{i}$, euclidean concepts will be employed (in the small), carried over from the $Y^{i_{-}}$ plane by the $Y^{i}$-projection (or, rather, its inverse). To avoid confusion, euclidean terminology, when thus employed, will be modified by the prefix $Y^{i}$. Thus, if $\Delta^{i}$ denotes any $m$-cell on $D^{i}$ whose $Y^{i}$-projection is a convex cell, then the $Y^{i}$-segment joining two points $(P, Q)$ on $\Delta^{i}$ means the arc on $\Delta^{i}$ whose $Y^{i}$-projection is the line-segment joining the projections of $P$ and $Q$. A $Y^{i}{ }^{i} r$-simplex similarly means an $r$-dimensional cell on $\Delta^{i}$, whose $Y^{i}$-projection is a simplex. Euclidean terms without prefixes have, of course, the usual meanings with reference to the euclidean metric of $E^{n}$.

It is not difficult to show that, for any $\epsilon>0$, however small, there exists a $\delta>0$ so small that if $P_{0}$ and $P_{1}$ are two distinct points on $D^{i}$ at distance less than $\delta$ apart, then $P_{0}$ and $P_{1}$ determine a $Y^{i}{ }^{i} 1-$ simplex, $\sigma_{1}$, whose tangent lines make angles less than $\epsilon$ with $P_{0} P_{1}$.

A set $\left(P_{0}, P_{1}, \cdots, P_{r}\right)$ of points on $D^{i}$ is called $Y^{i}$-independent if these points determine a nondegenerate $Y^{i}-r$-simplex, $\sigma_{r}$. They then also determine a nondegenerate rectilinear $r$-simplex, $s_{r}$, and are thus independent in the usual sense. The most obvious higher-dimensional analogue of the foregoing paragraph would appear to be a statement that if $s_{r}$ is of sufficiently small diameter, then each tangent $r$-plane to $\sigma_{r}$ makes an angle ${ }^{8}$ less than $\epsilon$ with the plane of $s_{r}$. This is, however,

7 The triangulation methods hereafter described can be carried out without any imbedding [10], using only the local euclidean geometries of a set of coordinate systems in terms of which $M$ is differentiable. However, the absolute euclidean geometry of $E^{n}$ is convenient and permits the incidental establishment of a number of important results.

${ }^{8}$ The cosine of such an angle is the inverse ratio of an $m$-dimensional volume on one plane to its projection on the other. 
false. For example, three points (however close together) on a great circle of a sphere determine a plane perpendicular to the sphere. However, the desired conclusion holds provided $s_{r}$ is bounded away from degeneracy; for example, following Whitehead, in terms of the relative thickness, $\tau\left(s_{r}\right)=\rho / l$, where $\rho$ is the distance from the centroid of $s_{r}$ to its boundary, and $l$ is the length of the longest edge of $s_{r}$.

(A) For any two positive constants $\epsilon$ and $\tau$, however small, there exists $a \delta>0$ so small that if the simplex determined by $\left(P_{0}, P_{1}, \cdots, P_{r}\right)$ is of thickness not less than $\tau$ and diameter not greater than $\delta$, then $\left(P_{0}, P_{1}, \cdots, P_{r}\right)$ determine a nondegenerate $Y^{i}-r$-simplex whose tangent $r$-plane at any point makes an angle less than $\epsilon$ with the plane of $s_{r}=P_{0} P_{1} \cdots P_{r}$.

The first proof $[7,10]$ that $M$ is triangulable consisted in spreading a triangulation over $M$ by a recurrent process, giving preference to $Y^{i}$-simplexes on $D^{i}(i=1, \cdots, N)$. The $Y^{i}$-simplexes for different values of $i$ were separated from one another by "cells of mixed straightness," which we proceed to describe.

Let $\Delta^{i j}$ be an $m$-cell on the intersection of $D^{i}$ and $D^{i}$, for some two different values of $i$ and $j$. Let $\left(P_{0}, P_{1}, \cdots, P_{r}\right)$ be a set of points satisfying the conditions of (A) above. Then $\left(P_{0}, P_{1}, \ldots, P_{r}\right)$ de-

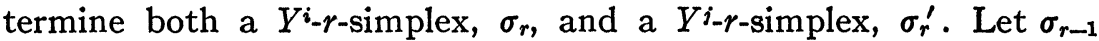
and $\sigma_{r-1}^{\prime}$ be the bounding $(r-1)$-cells of $\sigma_{r}^{\prime}$ and $\sigma_{r}^{\prime}$, respectively, opposite $P_{0}$.

(B) Under the conditions of (A) above, as the diameter of $s_{r}$ approaches zero, its relative thickness remaining bounded away from zero, $\sigma_{r-1}^{\prime}$ and $\sigma_{r-1}$ become increasingly good approximations to one another in such a

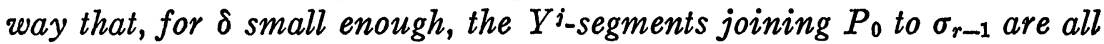
distinct and therefore determine a nondegenerate $r$-cell, $\sigma_{r}^{*}$. Since $\sigma_{r-1}$ is

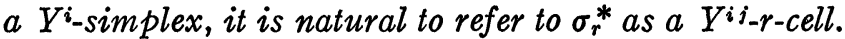

More generally, let $D^{i_{1}} \cdots i_{q}$ be the set of common inner points, assumed to be nonvacuous, of $D^{i_{1}}, \cdots, D^{i q}$.

Let $\sigma_{r-1} i_{1} \cdots i_{p}$ be a $Y^{i_{1}} \cdots i_{p_{-}}(r-1)$-cell on $D^{i_{1} \cdots i_{q}}$, assuming the term defined, for some $r$ of the set $(1, \cdots, m-1)$, where $p<r$ and $\left(i_{1}, \cdots, i_{p}\right)$ is a subset of $\left(j_{1}, \cdots, j_{q}\right)$. Let $P$ be a point on $\Delta^{i_{1}} \cdots i_{q}$, and let $j \neq\left(i_{1}, \cdots, i_{p-1}\right)$ be one of the numbers $\left(j_{1}, \cdots, j_{q}\right)$. If the $Y^{i}$-segments from $P$ to points of $\sigma_{r-1}{ }^{i_{1}} \cdots i_{p}$ are all distinct, and thus constitute a nonsingular $r$-cell $\sigma_{r}$, then $\sigma_{r}$ is called a $Y^{i_{1}} \cdots i_{p} j_{-} r$-cell or a $Y^{i_{1}} \cdots i_{p_{-}}-$-cell, according as $j \neq i_{p}$ or $j=i_{p}$. Commencing with an arbitrary point on $D^{i}$ as a $Y^{i}$-0-cell, we thus have a recurrent definition of $Y^{i_{1}} \cdots i_{p_{-}}$-cells.

(C) If, for a given $\tau<0,\left(P_{0}, P_{1}, \cdots, P_{r}\right)$ determine an $r$-simplex

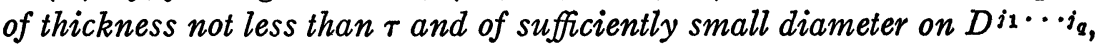


then $\left(P_{0}, P_{1}, \cdots, P_{r}\right)$ determine nondegenerate $Y^{i_{1}} \cdots i_{p-r}$-cells of all conceivable types, consistent with the definitions. In other words, the vertices $\left(P_{0}, P_{1}, \cdots, P_{r}\right)$ can be introduced, one at a time, in any order, in building up a simplicial cell as follows. With the first $\kappa$ vertices introduced $(\kappa \leqq r+1) k_{1}$-straightness can be employed for any $k_{1} \in\left(j_{1}\right.$, $\left.\cdots, j_{q}\right)$. If $\kappa \leqq r$, then with the next $\lambda$ points introduced, $\lambda \leqq r+1-\kappa$, $k_{2}$-straightness can be employed, where $\left(k_{1}, k_{2}\right)$ are any two different numbers of the set $\left(j_{1}, \cdots, j_{q}\right)$, and so on. This leads to the concept of " $\left(j_{1}, \cdots, j_{q}\right)$-independence" for a set such as $\left(P_{0}, P_{1}, \cdots, P_{r}\right)$.

8. A recurrent triangulation procedure $(m=2)$. In a lecture at the International Congress of Mathematicians at Zurich in 1932, Alexander [15] referred to the establishment of triangulation theorems for differentiable manifolds as "merely a matter of honest toil." It is indeed true that more patience than ingenuity was required to carry through the detailed modifications of rectilinear methods, which depend in a straightforward way on the facts that the metrics of two overlapping coordinate systems are continuously related and that the parallelism of one is a first approximation to that of the other. This "honest toil," a sort of mathematical pick-and-shovel work, was, however, a necessary task, if the combinatorial theory was to be directly applied to differentiable manifolds. Certain by-products of the labor, and certain of the methods employed, appear worthy of note and are presented below, free from the more tedious details.

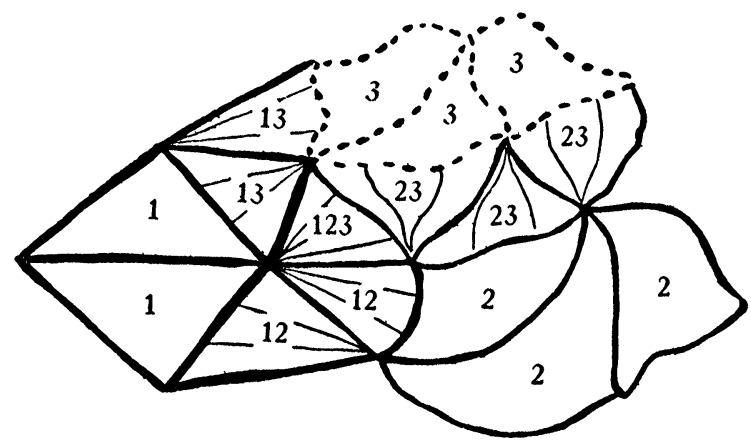

$Y^{i}$-simplexes and transitional cells in two dimensions

A brief outline of the most direct triangulation procedure will first be presented in the readily visualized case of a closed differentiable 2-manifold $M$ in $E^{3}$. The accompanying schematic diagram should be of assistance in following the steps of the process. Let $(y) \equiv\left(y_{1}\right.$, $\left.y_{2}, y_{3}\right)$ be rectangular cartesian coordinates. Using the notation of $\S 7$, let $Y^{1} \equiv\left(y_{2}, y_{3}\right), Y^{2} \equiv\left(y_{3}, y_{1}\right)$ and $Y^{3} \equiv\left(y_{1}, y_{2}\right)$. Then $D^{i}(i=1,2,3)$ 
is the region on $M$ where the direction cosine $\gamma^{i}$ exceeds $12^{-1 / 2}$. Let $D^{i i}$ denote the intersection of $D^{i}$ and $D^{i}(i \neq j)$, and let $D^{123}$ denote the common part of $D^{1}, D^{2}, D^{3}$. A convenient set of nonvacuous distinct regions covering $M$ is defined as follows:

$$
\begin{array}{lr}
D_{0}^{i}=D^{i}-\left(D^{i j}+D^{i k}\right) & ((i j k) \text { a permutation of }(123)), \\
D_{0}^{i j}=D^{i j}-D^{123} & (i \neq j), \\
D^{123} . &
\end{array}
$$

The closed regions $D_{0}^{i}(i=1,2,3)$ are bounded apart on $M$ by the open region $\left(D^{12}+D^{23}+D^{31}\right)$. Also, the subregions $D_{0}{ }^{12}, D_{0}{ }^{23}$, and $D_{0}^{31}$ of $D^{12}, D^{23}$, and $D^{31}$, respectively, are bounded apart on $\left(D^{12}+D^{23}+D^{31}\right)$ by $D^{123}$.

Let the $Y^{1}$-plane be triangulated into equilateral 2-simplexes $(c)^{\prime}$, and let $(\sigma)^{\prime}$ be the set of all $Y^{1}$-2-simplexes, plus bounding cells, each of which has one of the 2-simplexes $(c)^{\prime}$ for its $Y^{1}$-projection. The following subcomplexes of $(\sigma)^{\prime}$ will be used, it being understood in each case that all bounding cells of the specified 2-cells are included in the subcomplex.

$(\sigma)^{1}$ : those 2 -cells of $(\sigma)^{\prime}$ each having at least one vertex on the closure of $D^{1}-D^{12}$.

$(\tau)^{2}$ : those 2-cells of $(\sigma)^{\prime}$ entirely on $D^{12}$ and having no vertices in common with cells of $(\sigma)^{1}$.

$(\beta)^{12}$ : those 2 -cells not belonging to $(\sigma)^{1}$ or $(\tau)^{2}$ but having all their vertices on $(\sigma)^{1}+(\tau)^{2}$. Each 2-cell of this set has one or two of its vertices on $(\sigma)^{1}$ and the remaining vertex or vertices on $(\tau)^{2}$.

(A) The complex $(\sigma)_{0}^{\prime}=(\sigma)^{1}+(\beta)^{12}+(\tau)^{2}$ may not exhaust $(\sigma)^{\prime}$. There may, for example, be 2-simplexes in $(\sigma)^{\prime}$ lying entirely on $D^{12}$ and each having a vertex not belonging to either $(\sigma)^{1}$ or $(\tau)^{2}$. However, for any preassigned neighborhood $N^{1}$ of the boundary $\bar{D}^{1}-D^{1}$ of $D^{1}$, the complex $(c)^{\prime}$, which determines $(\sigma)^{\prime}$ and hence $(\sigma)_{0}^{\prime}$, can be made so fine that $(\sigma)_{0}^{\prime}$ will contain $D^{1}-N^{1}$.

(B) The fineness of $(c)^{\prime}$ will be restricted not only by the foregoing type of condition but also by the requirement that the vertices of each cell of $(\beta)^{12}+(\tau)^{2}$ be $Y^{12}$ independent $(\$ 7(\mathrm{C}))$.

As a consequence of $(B)$, it is possible to modify $(\sigma)_{0}^{\prime}$ by substituting for $(\tau)^{2}$ and $(\beta)^{12}$, respectively, the following complexes:

$(\sigma)_{0}{ }^{2}$ : the set of all $Y^{2}$-simplexes each determined by the vertices of one of the $Y^{1}$-simplexes $(\tau)^{2}$.

$(\sigma)^{12}$ : the set of all $Y^{12}$-2-cells, plus bounding cells, each determined by the vertices of one of the cells $(\beta)^{12}$ in such a way that $Y^{2}$-straightness prevails for bounding 1-cells with both vertices on 
$(\sigma)_{0}^{2}$ and $Y^{1}$-straightness prevails otherwise. (See the cells labeled $Y^{12}$ in the above diagram.)

(C) The complex $(\sigma)_{*}^{12}=(\sigma)^{1}+(\sigma)^{12}+(\sigma)_{0}^{2}$ has the same vertices as $(\sigma)_{0}^{\prime}$ and covers approximately the same region. In particular, the condition of statement (A) above can be assumed with $(\sigma)_{*}^{\mathbf{1 2}}$ in place of $(\sigma)_{0}^{\prime}$, since this condition can be assured by a further restriction, if necessary, on the fineness of $(c)^{\prime}$.

The introduction of the cells $(\sigma)_{0}^{2}$ facilitates a subdivision and extension of the triangulation $(\sigma)_{*}^{\mathbf{1 2}}$ to cover $D^{1}+D^{2}$, save for an arbitrarily restricted neighborhood, $N^{12}$, of the boundary of $D^{1}+D^{2}$. Let the $Y^{2}$-plane be triangulated into simplexes $(c)^{\prime \prime}$, and let $(\sigma)^{\prime \prime}$ denote the set of all $Y^{2}$-simplexes on $D^{2}$ each corresponding under the $Y^{2}$-projection to one of the simplexes of $(c)^{\prime \prime}$. Let $(\sigma)_{0}^{\prime \prime}$ denote the subcomplex of $(\sigma)^{\prime \prime}$ consisting only of those 2-cells thereof, plus bounding cells, which are free from points on $(\sigma)^{1}+(\sigma)^{12}$. Then $(\sigma)_{0}{ }^{\prime \prime}$ and $(\sigma)^{1}+(\sigma)^{12}+(\sigma)_{0}{ }^{2}$ overlap only on subcomplexes made up of $Y^{2}$-simplexes. Hence these complexes can be subdivided, using only $Y^{2}$-simplexes, so as to have in common exactly a subcomplex of both. There results a complex $(\sigma)_{* *}^{12}=(\sigma)^{1}+(\sigma)^{12}+(\sigma)^{2}$ which, by restrictions on the fineness of $(c)^{\prime}$ and $(c)^{\prime \prime}$, ean be required to cover all of $M$ save for the preassigned neighborhood $N^{12}$.

(D) The above work can be carried out $[7,10]$ in such a way as to ensure that the vertices of each cell of $(\sigma)_{* *}^{12}$ on $D^{i 3}(i=1,2)$ or $D^{123}$ satisfy the conditions of $\$ 7(\mathrm{C})$ for $(2,3)$-independence or $(1,2,3)$-independence. The details are here omitted.

It remains only to extend the triangulation $(\sigma)_{* *}^{12}$ over the remainder of $M$, which is a subset of $D^{3}$ and covers all save a neighborhood $N_{0}^{3}$ of $\bar{D}_{0}{ }^{3}$, where $N_{0}^{3}$ can be arbitrarily restricted in advance. The following subcomplexes of $(\sigma)_{* *}^{12}$ are useful, it being understood that bounding cells are included in each case:

$(\sigma)_{0}^{12}$ : the 2 -cells of $(\sigma)_{* *}^{12}$ each having at least one vertex on $D_{0}^{1}+D_{0}^{12}+D_{0}^{2}$.

$(\tau)^{3}$ : the subcomplex of $(\sigma)_{* *}^{12}$ consisting of all 2-cells thereof having no vertices in common with the subcomplex $(\sigma)_{0}{ }^{12}$.

$(\beta)$ : those 2-cells of $(\sigma)_{* *}^{12}$ which belong to neither $(\sigma)_{0}^{12}$ nor $(\tau)^{3}$.

As a consequence of (D), it is possible to modify $(\sigma)_{* *}^{12}$ by substituting for $(\tau)^{3}$ and $(\beta)$, respectively, the following complexes:

$(\sigma)_{0}^{3}$ : the set of all $Y^{3}$-simplexes each determined by the vertices of one of the simplexes of $(\tau)^{3}$.

$(\beta)^{18}+(\beta)^{23}+(\beta)^{123}$ : the $(\beta)^{i 3} \quad(i=1,2)$ are $Y^{i 3}$-cells determined by vertices of $Y^{i}$-simplexes of $(\beta)$ and the $(\beta)^{123}$ are $Y^{123}$-cells determined by vertices of $Y^{12}$-cells of $(\beta)$, it being understood that 
$Y^{3}$-straightness is to be used only relative to vertices on $(\tau)^{3}$.

The above description is intended to be suggestive rather than detailed.

(E) Let $(\sigma)_{*}=(\sigma)_{0}{ }^{12}+(\beta)_{0}{ }^{3}+(\beta)^{13}+(\beta)^{23}+(\beta)^{123}$. Then the boundary of $(\sigma)_{*}$ (and, with suitable restrictions, several "layers" of cells near the boundary) is made up exclusively of $Y^{3}$-simplexes. Since the remainder of $M$ is on $D^{3}$, it is easy to extend $(\sigma)_{*}$ into a complete triangulation of $M$ with the use of only $Y^{3}$-simplexes.

9. Generalization to higher dimensions. (A) The method outlined in $\$ 8$ generalizes directly to a recurrent process for spreading a triangulation over an entire differentiable m-manifold $M$ in $E^{n}$. After the jth step of the recurrency, the region $\left(D^{1}+D^{2}+\cdots D^{j}\right)$, save for an arbitrary preassigned neighborhood of its boundary, is covered by the triangulation, and all the cells are $Y^{i_{1}} \cdots i_{p_{-}}$cells, for various subsets $\left(i_{1}, \cdots, i_{p}\right)$ of $(1, \cdots, j)$. When the triangulation is extended over $D^{i+1}$, preference is given to $Y^{j+1}$-straightness, and the transition thereto is made with the aid of $Y^{i_{1}} \cdots i_{p}, j+1$-cells.

The whole process depends, as suggested by the results in $\$ 7$, on the existence of arbitrarily fine local subdivisions of $M$ which match up in a certain way and whose cells are bounded away from degeneracy in a manner not dependent on the fineness of the subdivision.

It should be noted that $Y^{i_{1}} \cdots i_{p_{-}}$cells are differentiable, insofar as interior points are concerned. However, they may have conical points (or loci of conical points) on their boundaries; as, for example, in the case of a 2-cell made up of straight segments from a point to a nonplanar differentiable arc in $E^{3}$. The presence of conical points is unimportant from the viewpoint of proving triangulability, but it is undesirable for a number of other reasons.

10. $C^{1}$-complexes. The piecemeal triangulation process of $\S \S 8$ and 9 was presented because of its directness. Whitehead's work, however, has the advantage of leading to a sort of "preferred class" of triangulations, referred to as $C^{1}$-triangulations or $C^{1}$-complexes, which resemble rectilinear complexes in possessing the basically important property of combinatorial equivalences, ${ }^{9}$ in the following sense.

(A) Any two $C^{1}$-triangulations of the same manifold have rectilinear models which possess isomorphic rectilinear subdivisions.

This property puts the topology of differentiable manifolds on a basis equivalent to that of euclidean polyhedra and provides a founda-

${ }^{9}$ This property is also shared by the writer's triangulations, by virtue of statement (B) below. However, it was not previously proved for them. 
tion for theorems involving both differential geometry and combinatorial topology.

A map $f(s)$ in $E^{n}$ of a $k$-simplex $s_{k}$ in $E^{k}$ is described as of class $C^{1}$ if it is continuously differentiable and can be extended in class $C^{1}$ fashion throughout some open neighborhood of $s_{k}$ in $E^{k}$. The map is referred to as nondegenerate if its jacobian is of rank $k$, and if the map can be extended over an open neighborhood of $s_{k}$ so as to preserve this property.

Let $K$ be a rectilinear simplicial complex in a euclidean space. Then $f(K) \subset E^{n}$ is called a $C^{1}$-map or a map of class $C^{1}$ if it is of class $C^{1}$ throughout each simplex of $K$. It is called nondegenerate if it is nondegenerate on each simplex. Such a map is referred to also as a $C^{1}$-complex. By virtue of this definition, the cells of a (nondegenerate) $C^{1}$-complex have no conical points on their boundaries.

The presence of conical points, noted at the end of $\S 9$, prevents the triangulations there described from being $C^{1}$-complexes. However, as Whitehead pointed out, a $C^{1}$-complex can be readily derived, as follows, from such a triangulation $(\sigma)$ of $M$ in $E^{n}$. Suppose, without further argument [19], that the vertices of each $m$-cell of $(\sigma)$ determine a nondegenerate $m$-simplex in $E^{n}$ and that the totality of such simplexes is a nonsingular inscribed polyhedron, $\Pi$, approximating to $M$ in $E^{n}$. (Such approximations are further discussed below.) Each face of $I I$ is assumed to make small angles with $M$ at its vertices. If $M$ is of class $C^{k}$ (but not of class $C^{k+1}$ ) in $E^{n}$, the normal $(n-m)$-planes to $M$ in $E^{n}$ form a system of class $C^{k-1}$. Whitney [40], however, with the aid of his analytic approximations, showed how to construct a class $C^{k}$ family of approximately normal $(n-m)$-planes.

(B) By means of a class $C^{k}$ family of $(n-m)$-planes approximately normal to $M$, an approximating polyhedron, $\Pi$, can be projected back into a triangulation $(\sigma)$ of $M$, where $(\sigma)$ is not merely a $C^{1}$-complex, but $a C^{k}$-complex. ${ }^{10}$

Whitehead [13] proved directly that an arbitrary differentiable manifold possesses a $C^{1}$-triangulation. His arguments, while in many respects refinements of the piecemeal triangulation of $\S \S 8$ and 9 , are sufficiently different to constitute an independent proof.

11. The associated approximation theory. Let $f(K)$ be a nondegenerate $C^{1}$-map of a rectilinear simplicial complex, $K$, in a euclidean space. Suppose (cf. $\$ 10$ ) that $f(K)=M$, where $M$ is a differentiable $m$-manifold in $E^{n}$. In other words, suppose $f(K)$ to be a $C^{1}$-triangula-

${ }^{10}$ The definition of a $C^{k}$-complex is an obvious modification of that of a $C^{1}$-complex. 
tion of $M$. Let $K^{\prime}$ be a subdivision of $K$, and let $f^{\prime}\left(K^{\prime}\right)$ be a $C^{1}$-map of $K^{\prime}$ in $E^{n}$. This map is described as an $(\epsilon, \rho)$-approximation to $f(K)$, if, throughout each simplex $s$ of $K^{\prime}$, we have

$$
\left\|f^{\prime}-f\right\| \leqq \epsilon \text { and }\left\|d f^{\prime}-d f\right\| \leqq \rho\|d f\|,
$$

where (1) $\|y\|$ stands for $\left(\sum_{i=1}^{n} y_{i}^{2}\right)^{1 / 2}$ and (2) $d f$ and $d f^{\prime}$ are tangent vectors to $f\left(K^{\prime}\right)$ and $f^{\prime}\left(K^{\prime}\right)$ respectively, corresponding to a vector $d x$ in $s$. If $f^{\prime}$ and $f$ agree, point for point, then $f^{\prime}\left(K^{\prime}\right)=f\left(K^{\prime}\right)$ is a $(0,0)$-approximation to $f(K)$ and represents a subdivision of the $C^{1}$-triangulation $f(K)$ of $M$.

The concept of an $(\epsilon, \rho)$-approximation can be applied in connection with a theory of polyhedral approximations to $M$. Let $(\sigma)$ denote the cells of the $C^{1}$-complex $f(K)$ covering $M$. An approximating euclidean polyhedron, $\Pi$, to $M$ can be obtained by replacing each of the cells $(\sigma)$ by the simplex in $E^{n}$ determined by its vertices. In the absence of further restrictions, there is no assurance that $I I$ will be nonsingular or that its faces will be nondegenerate.

The polyhedron $\Pi$, determined as just described by $f(K)=M$, can be represented by a map $L_{f}(K)=\Pi$, defined by the requirements that it be linear on each simplex of $K$ and coincide with $f$ at each of the vertices of $(\sigma)=f(K)$. The notation $L_{f}\left(K^{\prime}\right)=\Pi^{\prime}$ then represents a similarly defined piecewise linear map with reference to the subdivision $K^{\prime}$ of $K$. Thus $\Pi^{\prime}$ is, in a certain sense, a finer inscribed polyhedron than $\Pi$.

The subdivision $K^{\prime}$ of $K$ is called a $(\delta, \sigma)$-subdivision of $K$ if each simplex of $K^{\prime}$ is of diameter at most $\delta$ and of relative thickness at least $\sigma$.

(A) Given the map $f(K)$, two arbitrary positive constants $(\epsilon, \rho)$, and any positive thickness $\sigma$, there exists a $\delta>0$ so small that $L_{f}\left(K^{\prime}\right)=\Pi^{\prime}$ will be an $(\epsilon, \rho)$-approximation to $f(K)=M$ if $K^{\prime}$ is $a(\delta, \sigma)$-subdivision of $K$. Furthermore, if $(\epsilon, \rho)$ are small enough, then the $(\epsilon, \rho)$-approximation $\Pi^{\prime}$ to $M$ will be nonsingular.

The above result and the next following are due to Whitehead. Proofs [13] are here omitted. Taken together, these results lead to the form given below for the polyhedral approximation theorem.

(B) For an arbitrarily small $\delta>0$ and some fixed $\sigma>0, \sigma$ being independent of $\delta$, there exist $(\delta, \sigma)$-subdivisions of $K$.

Freudenthal's triangulation methods [12] employ an upper bound on the flatness of a simplex as a means of bounding it away from degeneracy, the flatness being defined as the ratio $l^{r} / v\left(s_{r}\right)$ where $v\left(s_{r}\right)$ is the $r$-dimensional volume of $s_{r}$ and $l$ is its longest edge. His theory included the use of arbitrarily fine simplicial subdivisions of 
limited flatness. Thus the rudiments, but not the development, of a polyhedral approximation theory were present. He employed a piecemeal method, analogous to that reported in $\S \S 8$ and 9 of this paper.

Now let $\delta$ take on successively the values $\delta^{\prime}, \delta^{\prime \prime}, \delta^{\prime \prime \prime}, \ldots$ in a sequence of positive numbers converging to zero, and let $\left(K^{\prime}, K^{\prime \prime}\right.$, $\left.K^{\prime \prime \prime}, \cdots\right)$ denote a sequence of $(\delta, \sigma)$-subdivisions of $K, \sigma$ remaining fixed while $\delta$ approaches zero (cf. (B) above). The successive polyhedra $\Pi^{\prime}=L_{f}\left(K^{\prime}\right), \Pi^{\prime \prime}=L_{f}\left(K^{\prime \prime}\right), \cdots$ then constitute a sequence of inscribed approximating polyhedra to $M$. In consequence of (A), the values $\left(\delta^{\prime}, \delta^{\prime \prime}, \ldots\right)$ can be chosen so that $\left(\Pi^{\prime}, \Pi^{\prime \prime}, \ldots\right)$ are, respectively, $\left(\epsilon^{\prime}, \rho^{\prime}\right)$-approximations, $\left(\epsilon^{\prime \prime}, \rho^{\prime \prime}\right)$-approximations, and so on, to $M$, where the $\epsilon$ 's and $\rho$ 's are preassigned sequences of positive numbers converging to zero.

Polyhedral APPRoximation theorem. The polyhedra $\left(\Pi^{\prime}, \Pi^{\prime \prime}\right.$, ...) constitute a sequence of inscribed polyhedral approximations to $M$. Under the homeomorphisms induced by the mapping $f(K)=M$ and the mappings $L_{f}\left(K^{\prime}\right)=\Pi^{\prime}, L_{f}\left(K^{\prime \prime}\right)=\Pi^{\prime \prime}, \cdots$, these polyhedra converge to $M$ as point sets. Their $m$-dimensional volumes also converge to the usual integral for the m-dimensional measure of $M$.

This theorem, stated for convenience in Whitehead's terminology, was established by the writer [19], using angles instead of relative thickness to keep the simplexes bounded away from degeneracy. It affords a direct generalization of the usual definition and formulation of arc length along a curve. As compared with examples revealing that any nonplanar differentiable surface in 3-space can be represented as the limit of a sequence of homeomorphic inscribed approximating polyhedra whose areas increase without limit instead of converging to the integral for surface area, the success of the present method depends on keeping the simplexes of the approximating polyhedra bounded away from degeneracy during the approximating process.

12. Some new results. The theorems stated in this section, while not previously published, are fairly direct and potentially useful consequences of the results outlined above. In the present discussion, details are avoided, but indications are given of method for constructing detailed proofs.

Let $M$ be an $m$-manifold of class $C^{k}(k>0)$ in $E^{n}$, and let $(y) \equiv\left(y_{1}\right.$, $\cdots, y_{n}$ ) be rectangular cartesian coordinates in $E^{n}$. Let $S$ be a set of local coordinate systems on $M$ such that (1) $M$ is of class $C^{k}$ in terms of $S$ and (2) the functions $y_{i}$ on $M$ are everywhere of class $C^{k}$ 
in terms of the systems $S$ and have a jacobian matrix of maximal rank $m$. In Whitney's paper [41] on imbedding manifolds in families of analytic manifolds, methods are employed whereby the systems $S$ can be augmented and extended as follows. Let $(u) \equiv\left(u_{1}, \cdots, u_{m}\right)$, with domain $\Delta$, be one of the systems $S$. In the terminology of Theorem I of Whitney's paper [41], $\Delta$ is an $m$-manifold (in particular, an $m$-cell) of class $C^{1}$ in "regular position" in $E^{n}$. It can, therefore, be imbedded in an $(n-m)$-parameter family, $\Delta\left(c_{1}, \cdots, c_{n-m}\right)$, of $m$-cells, in such a way that (1) $\Delta\left(c_{1}, \cdots, c_{n-m}\right)$ is analytic if $\left(c_{1}, \cdots, c_{n-m}\right) \neq 0$, (2) $\Delta(0, \cdots, 0)=\Delta,(3) \Delta\left(c_{1}, \cdots, c_{n-m}\right)$ is homeomorphic to $\Delta$, (4) tangential directions at corresponding points are approximately equal (see the original source for details), and (5) the cells $\Delta\left(c_{1}, \cdots, c_{n-m}\right)$ fill out, in a single-valued way, an open region $\Delta^{*}$ of $E^{n}$ containing $\Delta$. Then $(u)^{*} \equiv\left(u_{1}, \cdots, u_{m}\right.$, $\left.c_{1}, \cdots, c_{n-m}\right)$ can be regarded as a coordinate system with $\Delta^{*}$ for domain.

(A) Let each coordinate system ( $u$ ) of the set $S$ be augmented and extended, by the process just described, into a system (u)*. There then results a set $S^{*}$ of local coordinate systems in $E^{n}$ with the following properties: (1) Each of the systems $S^{*}$ is related to (y) by a transformation of class $C^{k}$ with nonvanishing jacobian, (2) the domains of the systems $S^{*}$ cover a neighborhood $N^{*}$ of $M$ in $E^{n}$, and (3) the part of $M$ on the domain of each local coordinate system appears as part of a coordinate m-plane, relative to that system.

Now let the whole space $E^{n}$ be regarded as an $n$-manifold of class $C^{k}$ in terms of the systems $S^{*}$ plus the system $(y)$, and let the piecemeal triangulation procedure described in $\$ \S 8$ and 9 be applied to $E^{n}$ in the following manner. The triangulation shall first be spread over the domains of systems in the set $S^{*}$, and then, as the last step of the recurrency, extended over the remainder of $E^{n}$. Consider any stage of the recurrency where the triangulation is being spread over the domain $\Delta^{*}$ of a system $(u)^{*}=\left(u_{1}, \cdots, u_{m}, c_{1}, \cdots, c_{n-m}\right)$. The recurrency involves a rectilinear simplicial subdivision of $\Delta^{*}$ (save for a neighborhood of its boundary) in terms of $(u)^{*}$-straightness. In this subdivision, we can require that the coordinate $m$-space of $\left(u_{1}, \cdots, u_{m}\right)$, which coincides with $M$ in the domain $\Delta^{*}$, be covered by a subcomplex. This property can be preserved throughout the entire recurrent process, until all the domains of the systems $S^{*}$ have been used. It will then be true that a neighborhood of $M$ is covered by a complex in which $M$ appears as a subcomplex. The final step of the recurrency, in which (1) a transition is made to straightness in terms of the metric of $E^{n}$ and (2) the subdivision is spread over the rest 
of $E^{n}$, does not affect any of the cells incident with $M$. Hence we have the following new theorem.

Theorem 12.1. If $M$ is an m-manifold of class $C^{k}$ in $E^{n}$, then the entire space $E^{n}$ can be triangulated into a $C^{k}$-complex ${ }^{11}$ in such a way that (1) $M$ coincides with a subcomplex of the triangulation and (2) all cells outside an arbitrary preassigned neighborhood of $M$ are rectilinear simplexes in the euclidean metric of $E^{n}$.

COROLLARY. It can be required that every cell of the triangulation of $E^{n}$ which has no vertices on $M$ shall be a rectilinear simplex.

For, once the triangulation is made, it can be arbitrarily finely subdivided, while the cells remain bounded away from degeneracy. The vertices of each cell can thus be made to determine a rectilinear simplex, such that the totality $(s)$ of these simplexes is a rectilinear simplicial subdivision of $E^{n}$. The subset $(s)^{0}$ of $(s)$ consisting of all simplexes thereof each having all its vertices on $M$ covers a polyhedral approximation, $\Pi$, to $M$ of the sort involved in the polyhedral approximation theorem in $\$ 11$. Now, using the process of the recurrency outlined in $\S \S 8$ and 9, let the cells $(s)^{0}$, and all cells incident with them, be modified so as to give precedence only on the cells $(s)^{0}$ to straightness in terms of the systems $S^{*}$. The corollary then follows directly.

THEOREM 12.2. Let $M^{r}$ be an r-manifold of class $C^{k}$ on an n-manifold $M^{n}$ of class $C^{k}(k>0)$. Then the entire manifold $M^{n}$ can be covered with a $C^{1}$-complex, a subcomplex of which covers $M^{r}$.

This theorem can be proved after the same fashion as Theorem 12.1. Local coordinate systems on $M^{n}$, whose domains cover a neighborhood of $M^{r}$, then play the role of the systems $(y)$ in $E^{n}$.

In the calculus of variations $[30,33]$ in the large, for example, the configuration of a differentiable $r$-manifold on a differentiable $n$-manifold is occasionally considered. It appears likely that Theorem 12.2 might prove useful in this connection; also in various other situations where submanifolds, chains or cycles are employed, and where it might prove convenient to regard them as subcomplexes of some covering triangulation.

Consider a closed differentiable $(n-1)$-manifold, $M^{n-1}$, in $E^{n}$, and let $\Pi^{n-1}$ be an inscribed approximating euclidean polyhedron, in the sense of the polyhedral approximation theorem in $\$ 11$. With the

${ }^{11}$ In the foregoing argument, some cells might have conical points. This can be avoided by an adaptation of Whitehead's methods. 
aid of a class $C^{1}$ family of approximately normal lines to $M^{n-1}$, it is possible to deform $E^{n}$ isotopically so that (1) all points remain fixed outside an arbitrary preassigned distance from $M^{n-1}$ and (2) $\Pi^{n-1}$ is carried into $M^{n-1}$. Hence we have the following theorem.

THEOREM 12.3. The regions into which $E^{n}$ is separated by $M^{n-1}$ are topologically equivalent to the regions into which $E^{n}$ is separated by a polyhedral approximation to $M^{n-1}$.

Corollary. A differentiable manifold in $E^{3}$ homeomorphic to a 2-sphere separates $E^{3}$ into two parts, the bounded one of which is a 3-cell.

The corollary follows with the aid of Alexander's proof ${ }^{12}$ of such a separation property for a euclidean polyhedron in $E^{3}$.

13. Applications. The divergence theorem and the generalized Theorem of Stokes offer the readiest illustrations of the use of cellular subdivisions in extending local results to results in the large. The simplest case, namely Green's Theorem in two dimensions, is outlined in $\$ 1$ above, and reference is there made to Kellogg's treatment [28] in $E^{3}$. The writer [18] proved the generalized theorem of Stokes for a differentiable orientable $r$-manifold $M^{r}$ with piecewise differentiable boundary on a differentiable $n$-manifold, $M^{n}$, shortly after establishing the necessary triangulation results. Previous proofs had applied only to a manifold $M$ in the space of a single coordinate system, where $M$ was either analytic or was subject to conditions not known to be fulfilled save by analytic manifolds. Let $Y_{i_{1}} \ldots i_{r-1}$ be an alternating tensor such that the partial derivatives $\partial Y_{i_{1}} \ldots i_{r-1} / \partial y_{i}$ are defined and continuous in a neighborhood of $\bar{M}^{r}$, where the $y^{\prime}$ s are a typical member of a set of local coordinates in terms of which $M^{n}$ is differentiable. Then the Stokes tensor of $Y_{i_{1}} \ldots i_{r-1}$ is defined as

$$
D_{i_{1} \ldots i_{r}}=\frac{1}{r !} \delta_{i_{1} \cdots i_{r}}^{\alpha_{1} \cdots \alpha_{r}} \frac{\partial Y_{\alpha_{2} \cdots \alpha_{r}}}{\partial y_{\alpha_{1}}}
$$

the summation convention holding for the $\alpha$ 's, and the $\delta$ 's being generalized Kronecker $\delta$ 's. If

$$
y_{i}=f_{i}\left(u_{1}, \cdots, u_{r}\right) \quad(i=1, \cdots, n)
$$

and

$$
y_{i}=g_{i}\left(v_{1}, \cdots, v_{r-1}\right) \quad(i=1, \cdots, n)
$$

are equations, respectively, of a typical $r$-cell of $M$ and of a typical

12 J. W. Alexander, Proc. Nat. Acad. Sci. U.S.A. vol. 10 (1924) pp. 6-8. 
$(r-1)$-cell of $B$, then Stokes' theorem can be formulated as the identity

$$
\begin{aligned}
\int_{M} \epsilon D_{\alpha_{1}} \cdots \alpha_{r} & \frac{\partial\left(y_{\alpha_{1}} \cdots y_{\alpha_{r}}\right)}{\partial\left(u_{1} \cdots u_{r}\right)} d u_{1} \cdots d u_{r} \\
= \pm \int_{B} \epsilon^{\prime} Y_{\alpha_{1}} \cdots \alpha_{r-1} & \frac{\partial\left(y_{\alpha_{1}} \cdots y_{\alpha_{r-1}}\right)}{\partial\left(v_{1} \cdots v_{r-1}\right)} d v_{1} \cdots d v_{r-1}
\end{aligned}
$$

where (1) the integrals are to be evaluated over the separate cells of the triangulation and the results summed, and (2) the $\epsilon$ 's are, on each cell, +1 or -1 according as the orientation of the cells by the parameters $(u)$ or $(v)$ agrees or disagrees with arbitrarily preassigned orientations of $M_{r}$ and $B_{r-1}$. The \pm in identity (13.4) depends on the relative orientations of $M_{r}$ and $B_{r-1}$. The integrals are absolute integral invariants under transformations of parameters and under transformations between coordinate systems. If summations are made, on both sides of (13.4), over all combinations of the $\alpha$ 's instead of being made as the $\alpha$ 's run independently from 1 to $n$, then the factor $r$ drops out.

The establishment of the theorem reduces, much as in the work of $\S 1$, to the problem of proving the identity for a typical cell of a triangulation and then summing over all the cells. For such a typical cell, the theorem of Stokes is obtained [18] with the aid of a class $C^{1}$ mapping, from the generalized divergence theorem as applied to an $m$-cell in euclidean $m$-space.

Hodge's work [27] includes (chap. 2) related material on the generalized theorem of Stokes. In general, Hodge's research on harmonic integrals was developed with the aid of local and global considerations pertaining to manifolds of class $C^{r}$. In this work, an interrelation is to be observed between topological properties and properties of differentiable geometry. The general fields of applicability described in Hodge's book appear to be essentially coextensive with those of the triangulation theorems here described. In both cases, the applications thus far made appear to be isolated and of a preliminary nature, relative to the possible applications, and one of the principle reasons for mentioning them is to call attention to the desirability of further investigations.

There is a considerable and growing body of literature to which reference should here be made. It would be impracticable to attempt an exhaustive bibliography, especially since the boundaries of this literature cannot easily be specified. However, reference should be made to contributions of Cartan [23], Chern [24-26], and de Rham 
[32]. Chern's papers, like Hodge's book, should be read not only for content, but for additional bibliographic references.

The homology groups of a manifold $M$ have proved important in a number of applications, including Hodge's theory of multiple integrals evaluated over chains of various dimensions and differentiability classes. Any covering complex on $M$ can serve to determine its homology groups. Algebraic geometry offers another field of application for homology theory and other aspects of combinatorial topology. This fact motivated van der Waerden's triangulation [1] of algebraic varieties. Other applications in algebraic geometry are due to Lefschetz $[3,29]$. In particular, one may note the adaptation to algebraic varieties of the multiplicity concept involved in the index of an intersection of two complexes. An agreement was revealed, with the aid of homology classes, between the topologic and algebraic concepts of multiplicity, in the sense that the algebraic multiplicity of an intersection of two algebraic manifolds having a finite number of points of intersection is equal to the topological index.

Further discussion of applications would be beyond the scope of the present paper. These fragmentary comments and the accompanying bibliography can do little more than serve as an initial guide to possible further study and research in the field. The same may be said of the material in the next section of this paper.

14. The general triangulation problem for manifolds and various ramifications thereof. In Alexander's lecture [15], already cited, the opinion was expressed that the triangulation and combinatorial equivalence problems reduce, for the general topological manifold, to showing the existence of an analytic (or a piecewise linear) homeomorphism approximating, with suitable restrictions, to an arbitrary topological mapping of an $n$-simplex in an $E^{n}$. This opinion is closely related to the following converse of the triangulation problem for differentiable manifolds, and also to Theorem 14.1 below. The attempt of Nöbeling [8] was based on analogous "smoothing" methods. Even in three dimensions, very difficult questions of a point theoretic nature are encountered.

THE REGULARITY PROBLEM. Let $M$ be an arbitrary topological mmanifold, and consider the set, $S_{0}$, of all coordinate systems whose domains are $m$-cells on $M$. The regularity problem is the problem of discovering whether $S_{0}$ contains any subset, $S_{1}$, in terms of which $M$ is of class $C^{1}$.

Since differentiable manifolds are triangulable, an affirmative solu- 
tion of the regularity problem would imply a solution of the triangulation problem for the general topological manifold. While the regularity problem thus presents a method of attacking the general triangulation problem for manifolds, it is possible that the regularity problem is too strong. In other words, there might possibly exist triangulable manifolds which cannot be made differentiable. This consideration suggested an investigation of the regularity problem not only for topological manifolds in general but for the much simpler case of triangulable manifolds. The following results were obtained.

TheORem 14.1. For manifolds of dimensionality less than 5 , the regularity problem is entirely equivalent to the triangulation problem. That is, on any triangulable m-manifold $M(m<5)$, there exists a set $S$ of coordinate systems in terms of which $M$ is differentiable.

THEOREM 14.2. If $M$ is a triangulable m-manifold $(m<5)$, then an analytic Riemannian geometry can be defined on $M$.

This follows from Theorem 14.1 with the aid of Whitney's results stated in $\$ 3$ above.

The articles [20-22] in which the above theorems were proved include a number of results bearing on the regularity problem for the general value of $m$. The mode of attack involves approximations. As in the case of the theorem affirming the topological equivalence of differentiable and analytic manifolds, the most promising approach appears to be with the aid of an imbedding in a euclidean space followed by the construction of a homeomorphic approximating manifold. In the present case, the given triangulable manifold is represented as a euclidean polyhedron, $\Pi^{m}$, in $E^{n}$. The writer's attempt, successful for $m<5$, to construct a differentiable $m$-manifold, $M$, approximating to $\Pi^{m}$ involved a study of spaces of $(n-m)$-planes transversal to $\Pi^{m}$. Transversality is a generalization of orthogonality. A connection is thus established with certain aspects of the study of sphere-spaces and fibre-spaces as developed by Whitney $[42,43]$, Stiefel [35] and others. In this connection, Chapter VIII of Lefschetz' Algebraic topology [29] is of interest and contains a number of references to related literature.

Theorem 14.2, with the triangulation theorem for the analytic case, establishes the topological equivalence of the classes of triangulable manifolds and analytic Riemannian manifolds. This suggests the possibility of interpreting, with respect to triangulable manifolds, theorems of topological content proved by methods of differential geometry. The Gauss-Bonnet theorem for Riemannian 
polyhedra, as treated by Allendoerfer and Weil [17], and also by Chern [25], appears relevant in this connection.

In closing, it may again be emphasized that the whole body of applications, and, in particular, the use of triangulation and approximation theorems to carry known results over to more general spaces, is largely a matter for future research. A mere beginning has been made.

\section{BIBLIOGRAPHY}

\section{Part A. Chronological list of Direct contributions to the TRIANGULATION PROBLEM}

1. B. L. van der Waerden, Topologische Begrïndung des Kalküls der abzählenden Geometrie, Anhang I, Math. Ann. vol. 102 (1929) pp. 360, 361.

2. S.S. Cairns, The cellular subdivision and approximation of regular spreads, Proc. Nat. Acad. Sci. U.S.A. vol. 16 (1930) pp. 488-491.

3. S. Lefschetz, Topology, Amer. Math. Soc. Colloquium Publications, vol. 12, 1930.

4. B. Koopman and A. B. Brown, On the covering of analytic loci by complexes, Trans. Amer. Math. Soc. vol. 34 (1932) pp. 231-251.

5. S. S. Cairns, On the cellular subdivision of $n$-dimensional regions, Ann. of Math. vol. 33 (1932) pp. 671-680.

6. S. Lefschetz and J. H. C. Whitehead, On analytic complexes, Trans. Amer. Math. Soc. vol. 35 (1933) pp. 510-517.

7. S. S. Cairns, On the triangulation of regular loci, Ann. of Math. vol. 35 (1934) pp. 579-587.

8. G. Nöbeling, Zur Topologie der Mannigfaltigkeiten, Monatshefte für Mathematik und Physik vol. 42 (1935) pp. 117-152.

9. H. Seifert, Review of Nöbeling's paper (preceding item), Zentralblatt für Mathematik und ihre Grenzgebiete vol. 11 (1935) p. 36.

10. S. S. Cairns, Triangulation of the manifold of class one, Bull. Amer. Math. Soc. vol. 41 (1935) pp. 549-552.

11. L. E. J. Brouwer, Zum Triangulationsproblem, Neder. Akad. Wetensch. vol. 42 (1939) pp. 701-706.

12. H. Freudenthal, Die Triangulation der differenzierbaren Mannigfaltigkeiten, Neder. Akad. Wetensch. vol. 42 (1939) pp. 880-901. See also Die Triangulation der differenzierbaren Mannigfaltigkeiten. Nachtrag., ibid. vol. 43 (1940) p. 619. 824.

13. J. H. C. Whitehead, On C1-complexes, Ann. of Math. vol. 41 (1940) pp. 809-

14. S. S. Cairns, Triangulated manifolds and differentiable manifolds, Lectures in topology, The University of Michigan Press, 1941, pp. 143-157.

\section{Part B. Partial list of Related literature}

15. J. W. Alexander, Some problems in topology, Verhandlungen des Internationalen Mathematiker Kongresses, Zurich, 1932, vol. 1, pp. 249-257.

16. P. Alexandroff and H. Hopf, Topologie I, Springer, 1935, chap. 3.

17. C. B. Allendoerfer, and A. Weil, The Gauss-Bonnet theorem for Riemannian polyhedra, Trans. Amer. Math. Soc. vol. 53 (1943) pp. 101-129. 
18. S. S. Cairns, The generalized theorem of Stokes, Trans. Amer. Math. Soc. vol. 40 (1936) pp. 167-174.

19. - Polyhedral approximations to regular loci, Ann. of Math. vol. 37 (1936) pp. 409-415.

20. - Homeomorphisms between topological manifolds and analytic manifolds, Ann. of Math. vol. 41 (1940) pp. 796-808.

21. - Isotopic deformations of geodesic complexes on the 2-sphere and on the plane, Ann. of Math. vol. 45 (1944) pp. 207-217.

22. - Introduction of a Riemannian geometry on a triangulable 4-manifold, Ann. of Math. vol. 45 (1944) pp. 218-219.

23. E. Cartan, La théorie des groupes finis et continus et la géométrie différentielle traitê par la méthode du repère mobile, Gauthier-Villars, 1937.

24. S. S. Chern, Integral formulas for the characteristic classes of sphere bundles, Proc. Nat. Acad. Sci. U.S.A. vol. 30 (1944) pp. 269-273.

25. - A simple intrinsic proof of the Gauss-Bonnet formula for closed Rieman. nian manifolds, Ann. of Math. vol. 45 (1944) pp. 747-752.

26. - Some new viewpoints in differentiable geometry in the large, Bull. Amer. Math. Soc. vol. 52 (1946) pp. 1-30.

27. W. V. D. Hodge, The theory and applications of harmonic integrals, Cambridge University Press, 1941, chap. 1.

28. O. D. Kellogg, Foundations of potential theory, Springer, 1929, chap. 4.

29. S. Lefschetz, Algebraic topology, Amer. Math. Soc. Colloquium Publications, vol. 27,1942 , especially chap. 8.

30. M. Morse, The calculus of variations in the large, Amer. Math. Soc. Colloquium Publications, vol. 18, 1934, chap. 5.

31. W. F. Osgood, Lehrbuch der Funktionentheorie I, Teubner, 1928, especially chap. 5 .

32. G. de Rham, Sur l'analysis situs des variétés à $n$ dimensions, Journal de Mathématique (9) vol. 10 (1931) pp. 115-200.

33. H. Seifert and W. Threlfall, Variationsrechnung im Grossen (Theorie von Marston Morse), Teubner, 1938.

34. H. A. Schwarz, Gesammelte mathematische Abhandlungen, Springer, 1890, vol. 2, pp. 309-311.

35. E. Stiefel, Richtungsfelder und Fernparallelismus in n-dimensionalen Mannigfaltigkeiten, Comment. Math. Helv. vol. 8 (1936) pp. 305-343.

36. W. Threlfall. See H. Seifert.

37. O. Veblen and J. H. C. Whitehead, The foundations of differential geometry, Cambridge Tracts, No. 29, Cambridge University Press, 1932.

38. A. Weil. See C. B. Allendoerfer.

39. J. H. C. Whitehead. See O. Veblen. 680.

40. H. Whitney, Differentiable manifolds, Ann. of Math. vol. 37 (1936) pp. 645-

41. - The imbedding of manifolds in families of analytic manifolds, Ann. of Math. vol. 37 (1936) pp. 865-877.

42. - Topological properties of differentiable manifolds, Bull. Amer. Math. Soc. vol. 43 (1937) pp. 785-805.

43. - On the topology of differentiable manifolds, Lectures in topology, Michigan, 1941, pp. 101-141.

Queens College, New York 